\title{
Acute and Chronic Cardioprotection by the Enkephalin Analogue, Eribis Peptide 94, is Mediated via Activation of Nitric Oxide Synthase and Adenosine Triphosphate-Regulated Potassium Channels
}

\author{
Garrett J. Gross $^{\mathrm{a}} \quad$ Anna Hsu ${ }^{\mathrm{a}} \quad$ Kasem Nithipatikoma $^{\mathrm{a}}$ Adam W. Pfeiffer \\ Irina Bobrova ${ }^{b}$ Erik Bissessar ${ }^{b}$ \\ aDepartment of Pharmacology and Toxicology, Medical College of Wisconsin, Milwaukee, Wisc., USA; \\ ${ }^{b}$ Eribis Pharmaceuticals AB, Uppsala, Sweden
}

\section{Key Words}

Opioid peptides $\cdot$ Myocardial infarction $\cdot$ Nitric oxide $\cdot \mathrm{K}_{\text {ATP }}$ channels

\begin{abstract}
Background/Aims: Eribis peptide 94 (EP 94) is a new enkephalin derivative which potently binds to the $\mu$ - and $\delta$-opioid receptor. In this study, we determined the effects of EP 94 and potential mechanism(s) involved in cardioprotection of the rat heart. Methods and Results: An acute (5 and 10 min into ischemia) and a chronic ( $24 \mathrm{~h}$ prior to ischemia) EP 94 administration produced a similar 30-40\% reduction in infarct size/area at risk and the effects were blocked by the $\mathrm{K}_{\text {ATP }}$ channel antagonists, HMR 1098 and 5-HD. The cardioprotective effects were blocked by a nonselective nitric oxide synthase (NOS) inhibitor (L-NAME) following acute administration and by a selective iNOS inhibitor (1400W) following chronic administration. Conclusion: These results suggest that EP 94 may have potential for the treatment of ischemic heart disease via a nitric oxide (NO)-K $\mathrm{K}_{\text {ATP }}-$ mediated mechanism.

Copyright $\odot 2012$ S. Karger AG, Basel
\end{abstract}

\section{Introduction}

The first evidence that opioids produced potent and efficacious cardioprotective effects via the activation of opioid receptors was published by Schultz et al. [1] in 1995. These experiments were performed on intact anesthetized rats in which the left coronary artery was occluded for $30 \mathrm{~min}$ of index ischemia followed by $2 \mathrm{~h}$ of reperfusion. Ischemic preconditioning (IPC) was used as a basis of comparison and was produced by either one or three 5-min periods of left coronary occlusion interspersed with $5 \mathrm{~min}$ of reperfusion prior to the $30-\mathrm{min}$ index ischemic period. IPC produced approximately a $70 \%$ reduction in infarct size (IS). Similarly, three 5-min infusions of the opioid agonist, morphine, at $100 \mu \mathrm{g} / \mathrm{kg}$, reduced the IS by approximately $65 \%$, similar to that of IPC. Interestingly, the effects of IPC and morphine were blocked by the ATP-sensitive potassium $\left(\mathrm{K}_{\mathrm{ATP}}\right)$ channel antagonist glibenclamide [2]. These data and several subsequent publications led to a surge of interest in studying the cardioprotective effects of opioids, primarily as they relate to their role in IPC and more recently postcondi-

\section{KARGER}

Fax +41613061234

E-Mail karger@karger.ch

www.karger.com
(C) 2012 S. Karger AG, Basel

0031-7012/12/0902-0110\$38.00/0

Accessible online at: www.karger.com/pha
Garrett J. Gross, $\mathrm{PhD}$

Department of Pharmacology and Toxicology

Medical College of Wisconsin, 8701 Watertown Plank Road

Milwaukee, WI 53226, USA

Tel. +1 414955 8627, E-Mail ggross@mcw.edu 
tioning [3], the receptors involved and their distal signaling pathways.

Eribis peptide 94 (EP 94) is a newly synthesized opioid peptide similar in structure to the naturally occurring enkephalins such as metenkephalin and leuenkephalin [4]. This compound has recently been shown to reduce myocardial IS in the rat heart and several pig models of ischemia/reperfusion injury by increasing the phosphorylation of eNOS (Ser 1177), suggesting an increase of eNOS activity and a subsequent release of nitric oxide $(\mathrm{NO})$ that is at least partially responsible for the acute cardioprotective effect of EP 94 in pigs. This is the first paper to demonstrate that opioids produce an increase of eNOS activity and NO release. Based on these intriguing results [5] and evidence that several NOS isoforms mediate cardioprotection [6-8], we extended these studies to determine the NOS isoforms that mediate the acute and chronic effects of EP 94 in a rat model of infarction. Our second objective was to determine the role of the sarcolemmal and mitochondrial $\mathrm{K}_{\mathrm{ATP}}$ channel in mediating the acute and chronic effect of EP 94 in reducing IS in the rat heart. This objective was based on our earlier studies where we demonstrated a role for both $\mathrm{K}_{\text {ATP }}$ channels in the cardioprotective effect of the synthetic opioid agonist, morphine, in isolated and intact rat hearts and isolated chick myocytes $[2,9,10]$. The results obtained suggest that $\mathrm{NO}$ and both $\mathrm{K}_{\mathrm{ATP}}$ channels are very important in mediating cardioprotection following EP 94 administration in rat hearts subjected to ischemia/ reperfusion.

\section{Methods}

Studies followed the Guide for the Care and Use of Laboratory Animals published by the United States National Institutes of Health (NIH Publications No. 85-23, revised 1996). The protocols were reviewed and approved by the Institutional Animal Care and Use Committee of the Medical College of Wisconsin.

\section{Materials}

EP 94 was obtained from Eribis Pharmaceuticals $A B$ (Uppsala, Sweden). N5-[imino(nitroamino)methyl]-L-ornithine, methyl ester, monohydrochloride (L-NAME) was obtained from Sigma-Aldrich Chemical (St. Louis, Mo., USA). (4S)-N-[4-amino-5(aminoethyl)aminopentyl]-N'-nitroguanidine (nNOS I) was obtained from EMD Millipore (Billerica, Mass., USA). N-[[3-(aminomethyl)phenyl]methyl]-ethanimidamide, dihydrochloride (1400W) and 5-hydroxydecanoate (5-HD) were obtained from Tocris Bioscience (Ellisville, Mo., USA). 1-\{[5-[2-(5-chloro- $o$-anisamido)ethyl]-2-methoxyphenyl]sulfonyl\}-3-methylthiourea, sodium salt (HMR 1098) was obtained from Aventis (Frankfurt, Germany). Rabbit polyclonal antibody against p-eNOS (Ser 1177) and eNOS, ECL Western blotting detection kit and BCA protein assay kit were from Pierce Biotechnology (Rockford, Ill., USA). Goat anti-rabbit IgG-HRP secondary antibody was obtained from Invitrogen (Camarillo, Calif., USA). Mini-PROTEAN gels were obtained from Bio-Rad Laboratories (Hercules, Calif., USA).

\section{In vivo Surgical Preparation}

Male Sprague-Dawley rats (250-300 g) were anesthetized with $100 \mathrm{mg} / \mathrm{kg}$ of Inactin and placed on a heating pad. A tracheotomy was performed and the rats were ventilated with room air supplemented with $100 \%$ oxygen (the $\mathrm{pO}_{2}$ was adjusted to approximately $150-200 \mathrm{~mm} \mathrm{Hg}$ ). Catheters were placed in the carotid artery and jugular vein for the measurement of systemic blood pressure, heart rate, blood gases and for the administration of drugs or vehicle. The heart was exposed by a left thoracotomy, and the left coronary artery was isolated and a suture was placed around it to produce an occlusion followed by reperfusion. The heart was subjected to $30 \mathrm{~min}$ of total coronary artery occlusion followed by $2 \mathrm{~h}$ of reperfusion at which time the artery was reoccluded and Evans blue dye was injected into the jugular vein to stain the nonischemic area of the heart blue. Subsequently, the heart was arrested with a bolus of $10 \% \mathrm{KCl}$ and removed for the measurement of IS. After the heart removal, it was washed in buffer and cut in a bread loaf fashion into 5-6 pieces. The blue-stained normal region and the ischemic region were separated and placed in separate vials containing triphenyltetrazolium chloride staining solution and incubated at $37^{\circ} \mathrm{C}$ in phosphate buffer at $\mathrm{pH} 7.4$ for approximately $15 \mathrm{~min}$. Subsequently, the pieces of tissue were placed into vials containing formaldehyde, stored overnight at room temperature and the IS was determined by planimetry the following day. The IS was expressed as a percent (\%) of the area at risk (IS/AAR, \%) as determined by our dual staining technique. In the AAR, the dark red color represents noninfarcted tissue, whereas, the infarcted tissue remains a pale yellow color.

\section{Experimental Protocols}

The various drugs and protocols are shown in figure 1 . Nineteen groups with 9 animals per group (total 171 rats) were subjected to the 30-min ischemia/2-hour reperfusion protocol. In all acute experiments, EP 94 was administered at 5 and 10 min into the ischemic period and the antagonists of the various isoforms of nitric oxide synthase (NOS) (L-NAME and nNOS I) and $\mathrm{K}_{\text {ATP }}$ channels (5-HD or HMR 1098) were administered 10 min prior to ischemia. In the chronic studies, EP 94 was administered $24 \mathrm{~h}$ prior to ischemia by intraperitoneal injection and the selective inducible NOS (iNOS) inhibitor, $1400 \mathrm{~W}$, or 5-HD or HMR 1098 were administered 10 min prior to ischemia. All drugs were dissolved in water in the original stock solutions, except for $1400 \mathrm{~W}$ which was dissolved in $95 \%$ ethanol and EP 94 which was dissolved in saline. They were then serially diluted in water to the desired concentrations and administered to the animals in water as a vehicle. 
Fig. 1. Schematic diagram of the various experimental protocols used in the present study. a Acute experimental protocol. b Chronic experimental protocol. $\mathrm{A}=$ Acute treatment; $\mathrm{C}=$ chronic treatment; $\mathrm{L}$ $\mathrm{NAME}=\mathrm{L}-\mathrm{N}^{\mathrm{G}}$-nitroarginine methyl ester; $1400 \mathrm{~W}=\mathrm{N}-[[3-($ aminomethyl $)$ phenyl $]$ methyl]-ethanimidamide; nNOS I = (4S)$\mathrm{N}$-[4-amino-5(aminoethyl)aminopentyl]N'-nitroguanidine; HMR = HMR 1098; 5-HD = 5-hydroxydecanoic acid; Isc = ischemia; Rep = reperfusion.

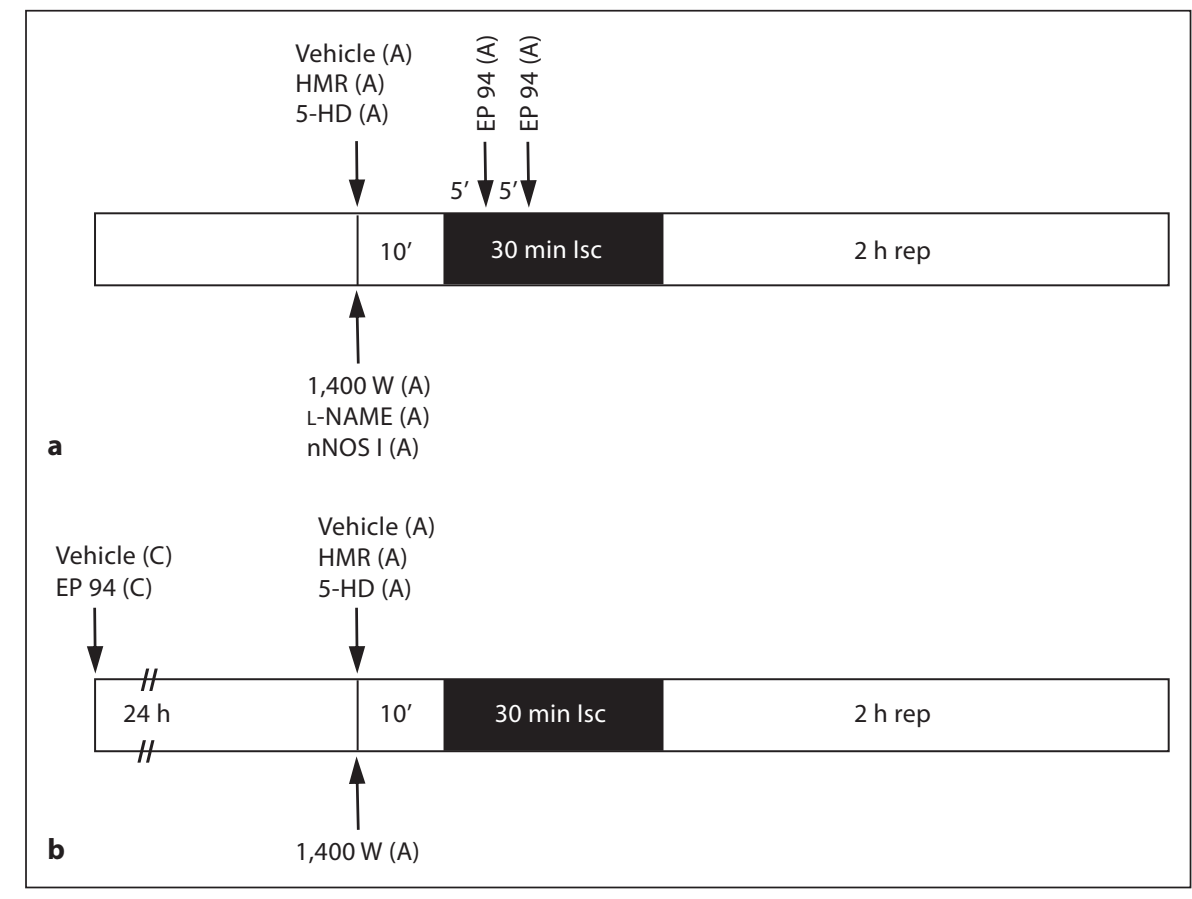

\section{Western Blot Analysis of p-eNOS (Ser 1177) in Rat Heart} Tissues

In this experiment, 3 rats were used for each group of treatment. Rat heart tissues (without staining) in the control rats (without ischemia/reperfusion), and nonischemic and ischemic areas of rat hearts subjected to $30 \mathrm{~min}$ of occlusion and $5 \mathrm{~min}$ of reperfusion were cut into pieces and put in sample tubes containing a protease and phosphatase inhibitor cocktail, snapped frozen in liquid nitrogen and stored at $-80^{\circ} \mathrm{C}$ until analysis. The frozen tissues were homogenized in the additional protease and phosphatase inhibitor cocktail and centrifuged at $10,000 \mathrm{rpm}$ at $4^{\circ} \mathrm{C}$ for $10 \mathrm{~min}$. The supernatant was determined for protein content using BCA protein assay kit and analyzed for expression of p-eNOS (Ser 1177) and total eNOS (t-eNOS) by Western blot analysis. Proteins were separated on SDS-PAGE (4-15\%) BioRad Mini-PROTEAN gels and transferred to a nitrocellulose membrane. Blots were incubated with p-eNOS (Ser 1177) primary antibody $(1: 1,000)$, t-eNOS primary antibody $(1: 1,000)$ or $\beta$-actin primary antibody $(1: 1,000)$ at $4^{\circ} \mathrm{C}$ overnight, followed by goat anti-rabbit IgG-HRP $(1: 50,000)$ at room temperature for 1 h. Protein loading, t-eNOS and $\beta$-actin were used as loading controls. Detection was made by using ECL Western blotting substrate and captured by Fuji film X-ray (Tokyo, Japan). Band densities were analyzed using Image J software from the NIH.

\section{Statistics}

Data were expressed as means \pm SEM. All data were analyzed using GraphPad Prism v.4.0. Comparison between groups in hemodynamics and blood gases was performed using a two- way ANOVA with post hoc analysis by the Bonferroni test. A one-way ANOVA was used to compare IS between groups followed by Tukey's post hoc test. A probability value of $p<0.05$ was considered statistically significant.

\section{Results}

\section{Exclusions}

A total of 178 rats were entered into the protocols shown in fig. 1. Seven rats were excluded due to intractable ventricular fibrillation (3 rats), abnormal blood gases ( 2 rats) and severe hypotension (mean arterial pressure less than $40 \mathrm{~mm} \mathrm{Hg}, 2$ rats). Therefore, 171 rats were included in the data analysis (19 groups of 9 rats).

\section{AAR/Left Ventricular Weight, Hemodynamics and Blood Gases}

There were no significant differences in the percent of AAR expressed as a percent of left ventricular weight (AAR/LV, \%) compared with the control group. There were no significant differences in the baseline (data not shown) values for heart rate and mean arterial pressure between all groups and at $30 \mathrm{~min}$ of ischemia and $2 \mathrm{~h}$ of reperfusion (data not shown). Similarly, there were no differences in blood gases or body temperature between groups throughout the experiment (data not shown). 


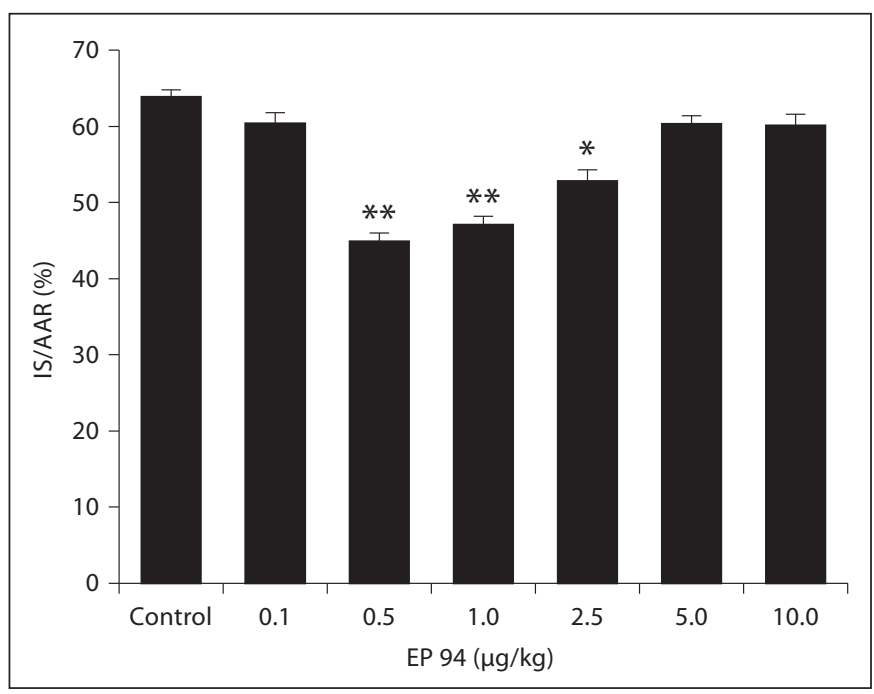

Fig. 2. Dose/response relationship and the reduction in IS/AAR (\%) produced by various doses of EP 94 . ( $n=9$ rats/group). ${ }^{\star} \mathrm{p}<$ 0.01 versus control; ${ }^{\star *} \mathrm{p}<0.001$ versus control.

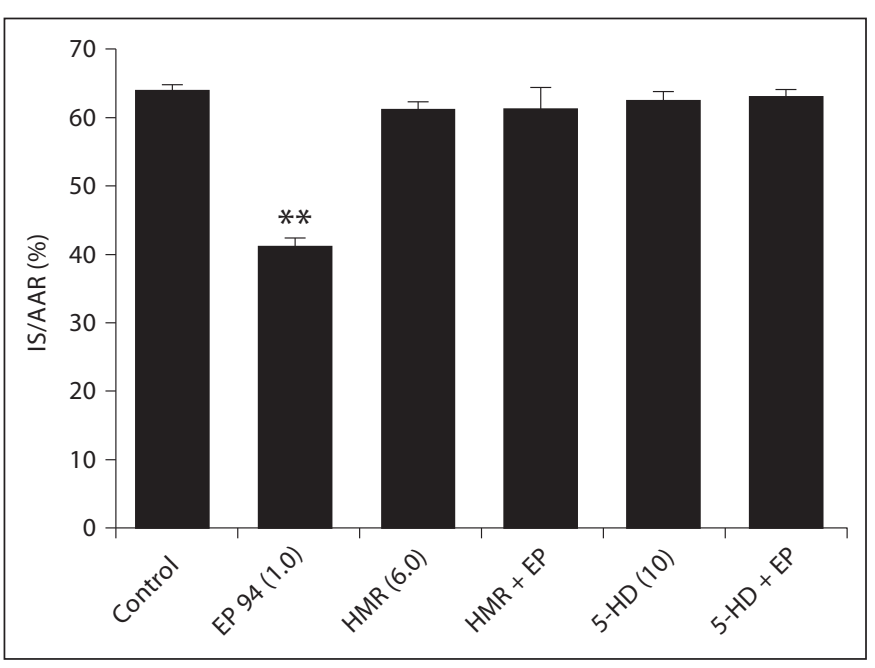

Fig. 3. Effects of the selective sarcolemmal (HMR 1098, $6 \mathrm{mg} / \mathrm{kg}$ i.v.) and the mitochondrial selective (5-HD, $10 \mathrm{mg} / \mathrm{kg}$ i.v.) $\mathrm{K}_{\mathrm{ATP}}$ channel antagonists on the IS-reducing effect of EP $94(1.0 \mu \mathrm{g} / \mathrm{kg}$ i.v.) in in vivo rat hearts ( $\mathrm{n}=9$ /group). ${ }^{\star \star} \mathrm{p}<0.001$ versus control.

\section{Dose/Response Studies}

In the initial series of experiments we performed a dose/response study using doses of EP 94 between 0.1 and $10 \mu \mathrm{g} / \mathrm{kg}$ i.v. EP 94 significantly reduced IS/AAR at $0.5,1.0$ and $2.5 \mu \mathrm{g} / \mathrm{kg}$ compared to the control group, with the maximal effect occurring at 0.5 and $1.0 \mu \mathrm{g} / \mathrm{kg}$ (fig. 2). Based on these data we chose the $1.0 \mu \mathrm{g} / \mathrm{kg}$ dose of EP

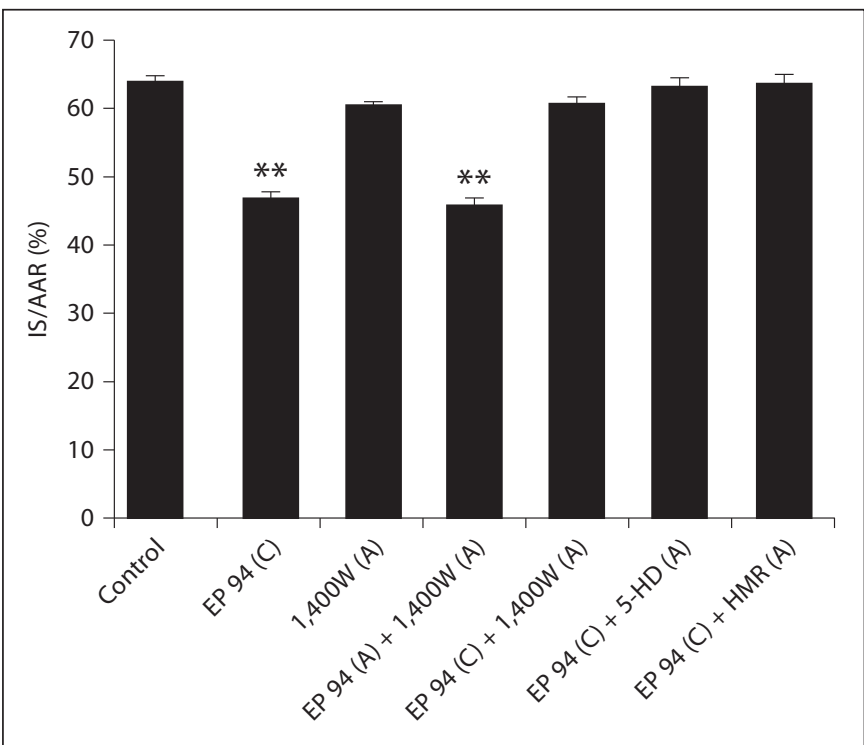

Fig. 4. Effect of EP 94 when administered $24 \mathrm{~h}$ prior to index ischemia and the subsequent effect of the selective iNOS inhibitor, $1400 \mathrm{~W}\left(0.1 \mathrm{mg} / \mathrm{kg}\right.$ i.v.) or the sarcolemmal or mitochondrial $\mathrm{K}_{\mathrm{ATP}}$ channel antagonists on the IS-reducing effect of EP $94(1.0 \mu \mathrm{g} / \mathrm{kg}$ i.v.) when given $10 \mathrm{~min}$ prior to index ischemia in rats previously treated with EP 94 for $24 \mathrm{~h}$ ( $\mathrm{n}=9$ /group). $\mathrm{A}=$ Acute; $\mathrm{C}=$ chronic. ${ }^{* *} \mathrm{p}<0.001$ versus control.

94 in all future experiments examining the role of $\mathrm{K}_{\mathrm{ATP}}$ channels and NO in EP 94-induced cardioprotection.

\section{Role of $K_{\text {ATP }}$ Channels in EP 94-Induced \\ Cardioprotection}

To investigate a possible downstream pathway which may be mediating the beneficial effect of acute EP 94, the sarcolemmal $\mathrm{K}_{\mathrm{ATP}}$ channel antagonist, HMR 1098, or the mitochondrial $\mathrm{K}_{\mathrm{ATP}}$ channel antagonist, 5-HD, were administered $10 \mathrm{~min}$ prior to ischemia. Both antagonists completely abolished the cardioprotective effect of EP 94 (fig. 3) which suggests an important role for the $\mathrm{K}_{\mathrm{ATP}}$ channels as a key mediator in the pathway by which EP 94 reduces IS similar to that seen with a number of other cardioprotective agents as well as IPC and postconditioning. In addition, both 5-HD and HMR 1098 blocked the cardioprotective effects of EP 94 following chronic EP 94 administration (fig. 4). These results were identical to those obtained in the acute EP 94 studies.

\section{Role of NO in Acute and Chronic EP 94-Induced Cardioprotection}

Finally, we investigated the roles of NO and eNOS in mediating the acute cardioprotection produced by EP 94, 


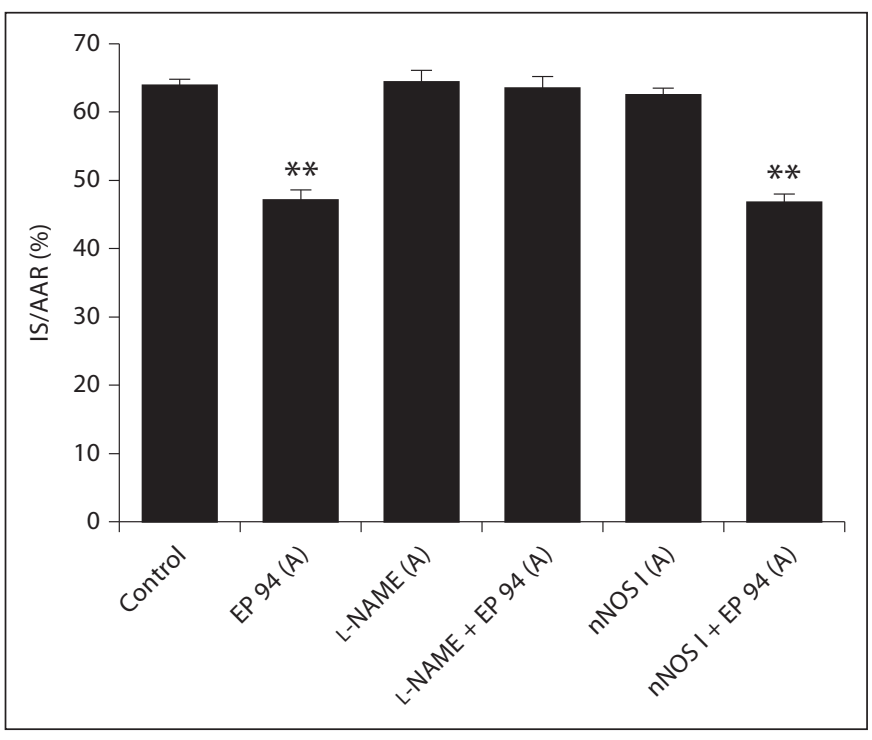

Fig. 5. The effect of two NOS inhibitors, L-NAME (nonselective NOS inhibitor) and nNOS I (selective neuronal NOS inhibitor) on the acute cardioprotective effect of EP $94(1.0 \mu \mathrm{g} / \mathrm{kg}$ i.v. $)$ in in vivo rat hearts ( $n=9$ /group). $A=$ Acute. ${ }^{\star *} \mathrm{p}<0.001$ versus control.

and the role of iNOS in mediating the chronic cardioprotection produced by EP 94 . The chronic administration of EP 94 produced a reduction in IS/AAR equal to that observed following its acute administration and this effect was blocked by $1400 \mathrm{~W}$, a selective iNOS inhibitor, administered $24 \mathrm{~h}$ after the EP 94 administration (fig. 4). Interestingly, $1400 \mathrm{~W}$ did not block the acute effect of EP 94 which is not surprising since eNOS is a more likely target for the early phase of protection. Our experiments with the nonselective NOS inhibitor, L-NAME, the selective nNOS inhibitor, nNOS I (fig. 5) and the selective inhibitor, $1400 \mathrm{~W}$, support a major role for eNOS in the acute cardioprotection produced by EP 94 . Both the selective nNOS and iNOS inhibitors did not block the protection produced by EP 94 acutely, whereas L-NAME, the nonselective NOS inhibitor, blocked the acute beneficial effect of EP 94 (figs. 4, 5).

\section{Effect of Ischemia/Reperfusion and EP 94 on the}

Phosphorylated-eNOS (Ser 1177)

Western blot analysis of rat heart tissues obtained from the control rats, and from nonischemic and ischemic areas of rat hearts subjected to $30 \mathrm{~min}$ of occlusion and 5 min of reperfusion with or without acute administration of EP $94(1.0 \mu \mathrm{g} / \mathrm{kg})$, showed an increase of the ratio of peNOS (Ser 1177)/t-eNOS. A 3-fold and a 2-fold increase of the ratio of p-eNOS (Ser 1177)/t-eNOS were observed in the ischemic and nonischemic areas, respectively (fig. 6a). Interestingly, EP 94 produced a 12-fold increase of the ratio of p-eNOS (Ser 1177)/t-eNOS in the ischemic areas of rat heart tissues and only about a 2 -fold increase in the nonischemic areas compared with the heart tissues of the control rats (fig. 6b). The intensity of the immunoreactive bands of $\mathrm{t}$-eNOS of the ischemic area tissues appeared to be higher than the control and nonischemic area tissues due to the protein loading as indicated by the band intensities of $\beta$-actin in these samples (fig. 6a, b).

\section{Discussion}

The results of the present study suggest that the enkephalin derivative, EP 94, possesses potent cardioprotective effects in reducing IS in an in vivo rat model of infarction. The optimal effect was observed at $0.5 \mu \mathrm{g} / \mathrm{kg}$ i.v. and its effect decreases with higher doses. The maximal effect was approximately a $30-40 \%$ reduction in IS expressed as a percent of the AAR. To prolong the plasma concentrations, EP 94 was administered as 2 boluses of $0.5 \mu \mathrm{g} / \mathrm{kg}$ i.v. at 5 and $10 \mathrm{~min}$ after the onset of the 30 -min index ischemia period. It is likely that EP 94 did not get to the heart during the ischemic period and its action probably occurs during the early reperfusion period. It would be of interest to determine if EP 94 is equally effective in rat hearts when administered just prior to reperfusion as suggested by recent data obtained in pig hearts [5], since this is the point where the heart is most susceptible to reperfusion-induced injury.

The magnitude of the effect of EP 94 in reducing IS/ AAR (\%) was equal to that seen with most opioid agonists studied in this model, including morphine [2] and TAN-67 [11], although EP 94 is one of the most potent compounds we have investigated in this model, if not the most potent. There were no noticeable effects of EP 94 on peripheral hemodynamics including heart rate and blood pressure. Similarly, no effects were observed on blood gases or body temperature throughout the experiments.

To investigate a possible downstream pathway which may be mediating the beneficial effect of EP 94, we administered the sarcolemmal $\mathrm{K}_{\text {ATP }}$ channel antagonist, HMR 1098, or the mitochondrial $\mathrm{K}_{\mathrm{ATP}}$ channel antagonist, 5-HD, prior to EP 94 administration. Both antagonists completely abolished the cardioprotective effect of EP 94. This suggests an important role for the $\mathrm{K}_{\mathrm{ATP}}$ channel as a key mediator in the pathway by which acute EP 94 reduces IS similar to that seen with a number of other 
Fig. 6. Western blot analysis of rat heart tissues obtained from nonischemic control rats, and from nonischemic and ischemic areas of rats subjected to $30 \mathrm{~min}$ occlusion and $5 \mathrm{~min}$ reperfusion. a Examples of immunoreactive bands of p-eNOS (Ser 1177) and t-eNOS of the control, and nonischemic and ischemic areas of rat heart tissues (left panel). Also shown are the immunoreactive bands of $\beta$-actin from the same membranes for $t$-eNOS. The average of the ratio of p-eNOS/t-eNOS as normalized to the control rat tissues (right panel; $\mathrm{n}=3$ rats). ${ }^{\star} \mathrm{p}<0.05$, significantly different from control. $\mathbf{b}$ Examples of immunoreactive bands of p-eNOS (Ser 1177) and $\mathrm{t}$-eNOS of the control (no treatment and occlusion and reperfusion), and ischemic and nonischemic areas of rats administered EP 94 (left panel). Also shown are the immunoreactive bands of $\beta$-actin from the same membranes for $\mathrm{t}$-eNOS. The average of the ratio of $\mathrm{p}$-eNOS/t-eNOS as normalized to the control rat tissues (right panel; $\mathrm{n}=3$ rats). Isc $=$ Ischemic. ${ }^{*} \mathrm{p}<0.05$, significantly different from control.

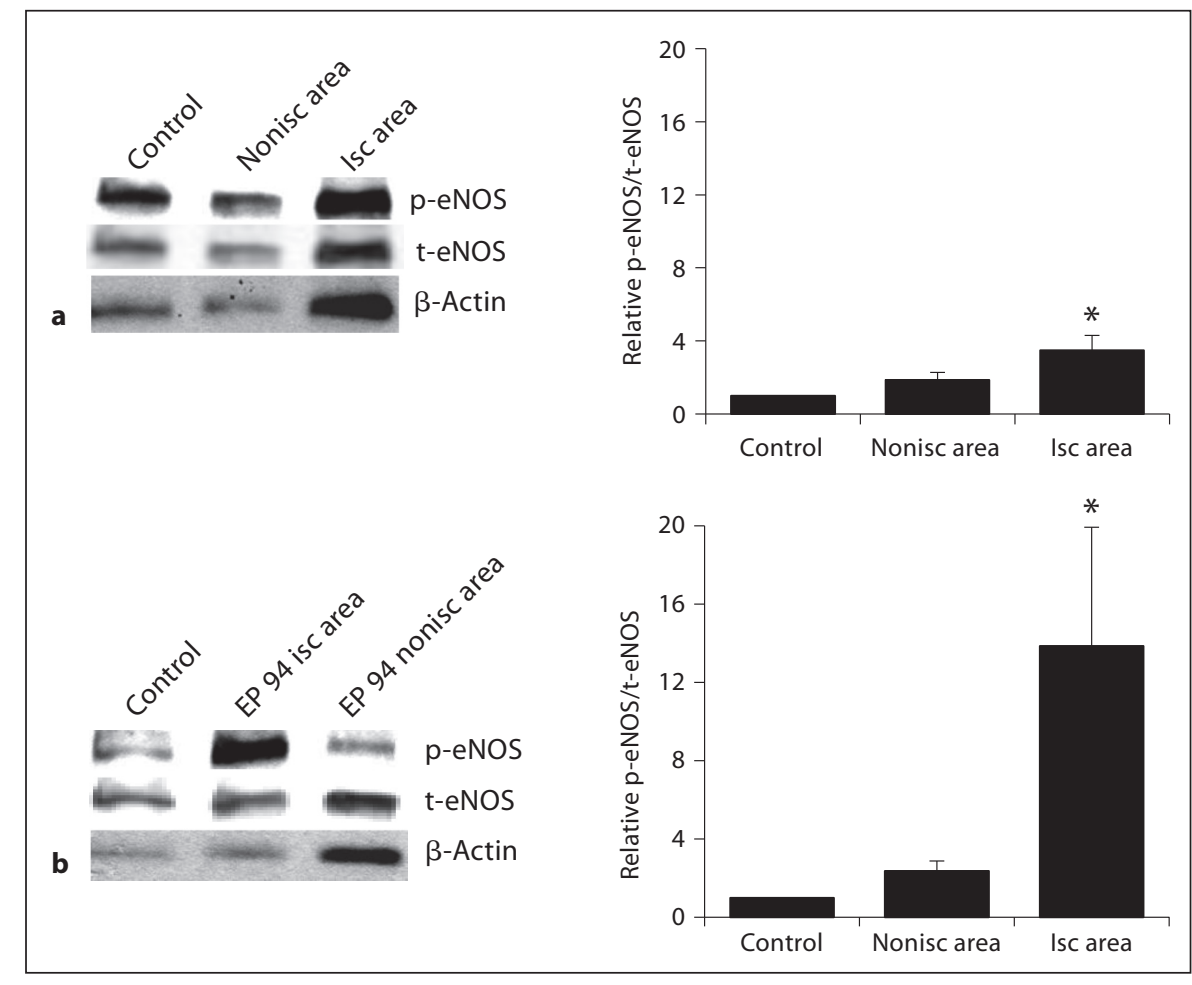

cardioprotective agents [12] as well as IPC and postconditioning [6-8]. Furthermore, both 5-HD and HMR 1098 blocked the delayed cardioprotective effect of EP 94 when administered $24 \mathrm{~h}$ after EP 94 treatment, which is similar to the data obtained in the acute experiments.

Finally, we investigated the ability and mechanism by which EP 94 produces the second window of cardioprotection $24 \mathrm{~h}$ after EP 94 administration and the role of NO and iNOS. We found that chronic EP 94 administration produced a reduction in IS/AAR equal to that observed following its acute administration and that this effect was blocked by a selective iNOS inhibitor, 1400W, administration $24 \mathrm{~h}$ after the EP 94 administration. These results suggest that EP 94 probably upregulated iNOS upon chronic exposure to EP 94. The detection of iNOS expression at various times during this chronic treatment protocol may provide direct evidence for this hypothesis. This finding that a drug (EP 94) produces an effect $24 \mathrm{~h}$ later similar in magnitude to when the drug was administered acutely is unique. Interestingly, 1400W did not block the acute effect of EP 94 which is not surprising since eNOS is a more likely target for the early phase of protection [5]. Similar results have been previously reported by Fryer et al. [13] and Jiang et al. [14] in rats and mice. These investigators found that the selec- tive $\delta_{1}$-opioid receptor agonist TAN-67 and morphine produced a second window of cardioprotection which was mediated by iNOS upregulation. Moreover, a selective inhibitor of nNOS, nNOS I, did not block the acute cardioprotective effect of EP 94 either but the nonselective NOS inhibitor, L-NAME, blocked the acute protective effect. Taken together, these data with selective and nonselective pharmacological blockers and Western blot analysis of p-eNOS (Ser 1177), clearly suggest that eNOS mediates the acute cardioprotective effect of EP 94 in the ischemic/reperfused rat heart, whereas iNOS mediates the chronic effect of EP 94. This is in agreement with the study by Shinmura et al. [8] and with previous work with other opioid agonists from our laboratory $[15,16]$ in rat and mouse hearts. Present results indicate that the NO release and $\mathrm{K}_{\mathrm{ATP}}$ channel openings are the downstream mediators in the acute cardioprotection produced by EP 94 , and the activation of a $\mu$-opioid receptor is likely involved [17]. It is of interest to understand the downstream signaling pathway(s) of NO involved in the cardioprotection by EP 94 administration acutely and chronically. The roles of NO in cardioprotection include the activation of Akt and extracellular receptor kinase (ERK) pathways as well as the peroxynitrite-matrix metalloproteinase- 2 signaling pathway in preconditioning [18-20]. 


\section{Conclusions}

In summary, the results of the present study suggest that the newly synthesized enkephalin derivative, EP 94, has a potent cardioprotective effect in reducing IS in a rat model of ischemia reperfusion injury. We also discovered that EP 94 exerts its beneficial effect by opening sarcolemmal and mitochondrial $\mathrm{K}_{\mathrm{ATP}}$ channels in the heart and has an acute effect in enhancing NO release most likely via eNOS phosphorylation in the heart. Of great interest is the novel observation that EP 94 exerts a cardioprotective effect $24 \mathrm{~h}$ after drug treatment via the upregulation of iNOS and enhanced NO release. Based on earlier results published by Frassdorf et al. [9], it is likely that the increase in iNOS activity results from the activation of the nuclear transcription factor, NF- $\kappa \mathrm{B}$, in rats. Future experiments are needed in animal models or humans to test this possibility. Nevertheless, EP 94 may be a novel new treatment for the acute and/or chronic treatment of patients with an acute coronary syndrome, particularly if its side-effect profile is less serious compared to standard treatment with opioids such as morphine.

\section{Acknowledgements}

These experiments were supported by funds obtained from Eribis Pharmaceuticals AB and NIH Grants HL074314 and HL111392 (G.J.G).

\section{References}

1 Schultz JE, Rose E, Yao Z, Gross GJ: Evidence for involvement of opioid receptors in ischemic preconditioning in rat hearts. Am J Physiol 1995;268:H2157-H2161.

2 Schultz JE, Hsu AK, Gross GJ: Morphine mimics the cardioprotective effect of ischemic preconditioning via a glibenclamidesensitive mechanism in the rat heart. Circ Res 1996;78:1100-1104.

-3 Zatta AJ, Kin H, Yoshishige D, Jiang R, Wang N, Reeves JG, Mykytenko J, Guyton RA, Zhao ZQ, Caffrey JL, Vinten-Johansen J: Evidence that cardioprotection by postconditioning involves preservation of myocardial opioid content and selective opioid receptor activation. Am J Physiol Heart Circ Physiol 2008;294:H1444-H1451.

4 BarronBA:Cardiacopioids. ProcSocExpBiol Med 2000;224:1-7.

5 Karlsson LO, Grip L, Bissessar E, Bobrova I, Gustafsson T, Kavianipour M, Odenstedt J, Wikstrom G, Gonon AT: Opioid receptor agonist Eribis peptide 94 reduces infarct size in different porcine models for myocardial ischaemia and reperfusion. Eur J Pharmacol 2011;651:146-151.

-6 Burkard N, Williams T, Czolbe M, Blomer N, Panther F, Link M, Fraccarollo D, Widder JD, Hu K, Han H, Hofmann U, Frantz S, Nordbeck P, Bulla J, Schuh K, Ritter O: Conditional overexpression of neuronal nitric oxide synthase is cardioprotective in ischemia/reperfusion. Circulation 2010;122: 1588-1603.
7 Jones SP, Greer JJ, Kakkar AK, Ware PD, Turnage RH, Hicks M, van Haperen R, de Crom R, Kawashima S, Yokoyama M, Lefer DJ: Endothelial nitric oxide synthase overexpression attenuates myocardial reperfusion injury. Am J Physiol Heart Circ Physiol 2004; 286:H276-H282.

-8 Shinmura K, Xuan YT, Tang XL, Kodani E, Han H, Zhu Y, Bolli R: Inducible nitric oxide synthase modulates cyclooxygenase- 2 activity in the heart of conscious rabbits during the late phase of ischemic preconditioning. Circ Res 2002;90:602-608.

$>9$ Frassdorf J, Weber NC, Obal D, Toma O, Mullenheim J, Kojda G, Preckel B, Schlack W: Morphine induces late cardioprotection in rat hearts in vivo: the involvement of opioid receptors and nuclear transcription factor kappaB. Anesth Analg 2005;101: 934-941.

10 Liang BT, Gross GJ: Direct preconditioning of cardiac myocytes via opioid receptors and KATP channels. Circ Res 1999;84:1396-1400.

-11 Gross ER, Gross GJ: Ischemic preconditioning and myocardial infarction: an update and perspective. Drug Discov Today Dis Mech 2007;4:165-174.

12 Gross ER, Gross GJ: Pharmacologic therapeutics for cardiac reperfusion injury. Expert Opin Emerg Drugs 2007;12:367-388.

13 Fryer RM, Wang Y, Hsu AK, Gross GJ: Essential activation of PKC-delta in opioid-initiated cardioprotection. Am J Physiol Heart Circ Physiol 2001;280:H1346-H1353.
14 Jiang X, Shi E, Nakajima Y, Sato S: Inducible nitric oxide synthase mediates delayed cardioprotection induced by morphine in vivo: evidence from pharmacologic inhibition and gene-knockout mice. Anesthesiology 2004; 101:82-88.

-15 Fryer RM, Hsu AK, Eells JT, Nagase H, Gross GJ: Opioid-induced second window of cardioprotection: potential role of mitochondrial KATP channels. Circ Res 1999;84: 846-851.

16 Patel HH, Hsu AK, Gross GJ: Cox-2 and iNOS in opioid-induced delayed cardioprotection in the intact rat. Life Sci 2004;75:129-140.

17 Gross GJ, Hsu A, Nithipatikom K, Bobrova I, Bissessar E: Eribis peptide 94 reduces infarct size in rat hearts via activation of centrally located $\mu$-opioid receptors. J Cardiovasc Pharmacol 2012;59:194-197.

18 Caimmi PP, Molinari C, Uberti F, Micalizzi E, Valente G, Mary DA, Vacca G, Grossini E: Intracoronary levosimendan prevents myocardial ischemic damages and activates survival signaling through ATP-sensitive potassium channel and nitric oxide. Eur J Cardiothorac Surg 2011;39:e59-e67.

19 Peart JN, Hoe LE, Gross GJ, Headrick JP: Sustained ligand-activated preconditioning via $\delta$-opioid receptors. J Pharmacol Exp Ther 2011;336:274-281.

20 Bencsik P, Kupai K, Giricz Z, Görbe A, Pipis J, Murlasits Z, Kocsis GF, Varga-Orvos Z, Puskás LG, Csonka C, Csont T, Ferdinandy $\mathrm{P}$ : Role of iNOS and peroxynitrite-matrix metalloproteinase-2 signaling in myocardial late preconditioning in rats. Am J Physiol Heart Circ Physiol. 2010;299:H512-H518. 